INDO GLOBAL JOURNAL OF

PHARMACEUTICAL SCIENCES

ISSN 2249- 1023

\title{
Exploitation of a Local Isolate, Brevibacillus Invocatus MTCC 9039 for Production of Poly(3-hydroxybutyrate-co-3- hydroxyvalerate) co-polymer
}

\author{
Indu Singh Sankhla ${ }^{1^{*}}$, Akhilesh Kumar Singh ${ }^{2}$, Nirupama Mallick ${ }^{3}$ \\ ${ }^{1}$ Department of Botany, University of Rajasthan, Jaipur-302004, Rajasthan, India \\ ${ }^{2}$ Amity Institute of Biotechnology, Amity University, Uttar Pradesh, Lucknow-226010, India \\ ${ }^{3}$ Agricultural \& Food Engineering Department, Indian Institute of Technology Kharagpur-721302, West Bengal, India
}

Address for Correspondence: Indu Singh Sankhla; induiitkgp@gmail.com

\begin{abstract}
A local isolate, identified as Brevibacillus invocatus with an accession number MTCC 9039 at Microbial Type Culture Collection (MTCC), Institute of Microbial Technology, Chandigarh, India, was validated as the producer of a poly(3-hydroxybutyrate-co-3-hydroxyvalerate) [P(3HB-co-3HV)] co-polymer. Synthesis of $\mathrm{P}(3 \mathrm{HB}-$ co-3HV) co-polymer under batch mode was investigated under supplementation of glucose, acetate and propionate. Under controlled condition, B. invocatus MTCC 9039 cells harvested at the stationary phase of growth registered maximum production of poly-3-hydroxybutyrate (PHB), i.e., $3 \%$ of dry cell weight (dcw) at $\mathrm{pH} 7.0$ and temperature $30^{\circ} \mathrm{C}$ at 48 hours of incubation. The PHB synthesis was boosted up to $52 \%$ (dcw) with the supplementation of $3 \%$ glucose and $1 \%$ acetate. P(3HB-co-3HV) co-polymer production was stimulated under propionate supplementation. This production was boosted up to $45 \%$ under 3\% glucose with $1 \%$ propionate supplementation. Optimization of process parameters by response surface methodology (RSM) further boosted the co-polymer production up to $65 \%(\mathrm{dcw})$ at $2.08 \%$ glucose, $1.62 \%$ acetate, $0.75 \%$ propionate and $2.15 \mathrm{gl}^{-1}$ $\mathrm{KH}_{2} \mathrm{PO}_{4}$ concentrations. This co-polymer not only depicted comparable material properties with the commercial $\mathrm{P}(3 \mathrm{HB}$-co3HV) co-polymers but also displayed the elasticity, which was immensely high and could be comparable with polypropylene, therefore advocating its potential applications in various fields. Hence, B. invocatus MTCC 9039 is emerging as an attentiongrabbing organism that could be further exploited for P(3HB-co-3HV) co-polymer production. (C) 2014 iGlobal Research and Publishing Foundation. All rights reserved.
\end{abstract}

Conference Proceedings: International Conference on Life Sciences, Informatics, Food and Environment; August 29- 30, 2014

Indo Global Journal of Pharmaceutical Sciences( ISSN 22491023 ; CODEN- IGJPAI; NLM ID: 101610675) indexed and abstracted in EMBASE(Elsevier), SCIRUS(Elsevier),CABI, CAB Abstracts, Chemical Abstract Services(CAS), American Chemical Society(ACS), Index Copernicus, EBSCO, DOAJ, Google Scholar and many more. For further details, visit 